\title{
Induction of human leukemia U937 cell apoptosis by an ethanol extract of Dendropanax morbifera Lev. through the caspase-dependent pathway
}

\author{
JOON WOO LEE ${ }^{1 *}$, CHEOL PARK $^{2 *}$, MIN HO HAN ${ }^{3}$, SU HYUN HONG $^{4}$, TAE KYUNG LEE ${ }^{5}$, \\ SHIN HWA LEE ${ }^{6}$, GI-YOUNG KIM ${ }^{7}$ and YUNG HYUN CHOI ${ }^{3,4}$
}

\author{
${ }^{1}$ Bunpo School, Nam-gu, Busan 608-832; ${ }^{2}$ Department of Molecular Biology, College of Natural Sciences, \\ Dongeui University, Busan 614-714; ${ }^{3}$ Anti-Aging Research Center and Blue-Bio Industry RIC, Dongeui University, \\ Busan 614-714; ${ }^{4}$ Department of Biochemistry, Dongeui University College of Oriental Medicine, Busan 614-052, \\ Republic of Korea; ${ }^{5}$ Department of Biology, New York University, New York, NY 10012, USA; \\ ${ }^{6}$ Department of Biological Sciences, Korea Advanced Institute of Science and Technology, Daejeon 305-701; \\ ${ }^{7}$ Laboratory of Immunobiology, Department of Marine Life Sciences, Jeju National University, \\ Jeju 690-756, Republic of Korea
}

Received April 19, 2013; Accepted June 3, 2013

DOI: $10.3892 /$ or.2013.2542

\begin{abstract}
Dendropanax morbifera Leveille is found throughout southwestern Korea, and has been used in traditional medicine for various diseases, such as migraine headache, infectious diseases, skin diseases and dysmenorrhea. However, the molecular mechanisms of D. morbifera concerning its biochemical actions in cancer have not yet been clearly elucidated. In the present study, we investigated the pro-apoptotic effects of an ethanol extract of D. morbifera stem bark (EEDM) on human leukemia U937 cells. EEDM markedly inhibited the growth of U937 cells by decreasing cell proliferation and inducing apoptosis. EEDM-induced apoptosis in U937 cells was associated with the upregulation of death receptor-related protein levels and downregulation of anti-apoptotic IAP family proteins. The increase in apoptosis was also associated with proteolytic activation of caspase- $8,-9$ and -3 , inhibition of anti-apoptotic Bcl-2 and Bcl-xL expression, Bid cleavage, and loss of MMP suggesting that apoptosis of U937 cells induced by EEDM was through the extrinsic and intrinsic pathways. However, a pan-caspase inhibitor, z-VED-fmk, significantly inhibited EEDM-induced U937
\end{abstract}

Correspondence to: Dr Yung Hyun Choi, Department of Biochemistry, Dongeui University College of Oriental Medicine, Yangjung-dong San 45, Busanjin-gu, Busan 614-052, Republic of Korea

E-mail: choiyh@deu.ac.kr

${ }^{*}$ Contributed equally

Key words: Dendropanax morbifera Leveille, U937 cells, caspase, PI3K/Akt, MAPKs cell apoptosis indicating that the caspases were key regulators of apoptosis in response to EEDM in U937 cells. Our data suggest that $D$. morbifera may be a potential anticancer agent for cancer treatment.

\section{Introduction}

Apoptosis is a form of programmed cell death defined by a characteristic set of morphological and biochemical changes that occurs during embryonic development and tissue homeostasis and involves eliminating unwanted or damaged cells from eukaryotic organisms. Apoptosis is mediated by either the extrinsic or intrinsic pathway, and both pathways converge on the activation of effector caspases, a family of cysteine-dependent aspartate-directed proteases, by initiator caspases $(1,2)$. In the extrinsic apoptosis pathway, interaction between ligands and death receptors initiates the pathway at the plasma membrane and subsequently activates initiator caspases such as caspase- 8 . Caspase- 8 can directly activate downstream effector caspases, including caspase-3 $(3,4)$. In the intrinsic apoptosis pathway, the mitochondrial membrane potential (MMP) is altered, and the $\mathrm{B}$ cell lymphoma $2(\mathrm{Bcl}-2)$ family of proteins plays a central role in regulating cell death. This process leads to the release of pro-apoptotic proteins from the mitochondrial intermembrane space, induces activation of caspase-9, and subsequently activates effector caspases including caspase-3 (5-7). After caspase-3 is activated, several specific substrates are cleaved or other cellular proteins are activated, eventually leading to apoptosis (8-10). In some cells, caspase- 8 also mediates the intrinsic pathway via cleavage of the pro-apoptotic Bid, a BH3-only protein $(2,11,12)$. Because aberrant regulation of apoptosis is representative of cancer and many therapeutic agents eliminate tumor cells by inducing apoptotic cell death, induction of apoptotic cell death is an important mechanism of action of potential anticancer drugs. 
Natural products, including plants, microorganisms, and marine organisms, are valuable sources of therapeutic compounds. This is particularly evident in cancer therapy, in which more than $60 \%$ of approved drugs are of natural origin (13). Therefore, a new natural source of anticancer compounds that have relatively fewer side-effects would be a valuable tool in cancer therapy. In particular, traditional medicine has been the focus of scientific efforts to discover novel anticancer agents. Although Oriental medicine has been used clinically in Asia, including Korea, for thousands of years as an important alternative remedy for cancer treatment, the molecular mechanisms underlying the effects of these natural products obtained from the extracts of medicinal plants are still unclear. Dendropanax morbifera Leveille is a subtropical, broad-leaved evergreen tree belonging to the family Araliaceae endemic to southwestern Korea. The roots, leaves, seeds and stems of this plant have been widely used in Korea as folk medicine for treating headache, infectious diseases, skin diseases, and other maladies. Recently, D. morbifera has been shown to possess potent anti-diabetic (14), anti-atherogenic (15), anti-plasmodial (16) and anti-complement activity $(17,18)$. In addition, studies on the anti-inflammatory and anticancer effects of components isolated from this plant also have been reported (19-21); however, the molecular mechanisms of its pro-apoptotic action in cancer cells are not fully understood.

Thus, in this study, as part of our ongoing screening program to evaluate the anticancer potential of $D$. morbifera, we investigated the pro-apoptotic property of an ethanol extract of D. morbifera stem bark and the responsible underlying molecular mechanisms of action in human leukemia U937 cells.

\section{Materials and methods}

Reagents. 3-(4,5-Dimethylthiazol-2-yl)-2,5-diphenyltetrazolium bromide (MTT), propidium iodide (PI), 4,6-diamidino-2-phenylindole (DAPI), and 5,5',6,6'-tetrachloro-1,1',3,3'-tetraethyl-imidacarbocyanine iodide (JC-1) were purchased from Sigma-Aldrich (St. Louis, MO, USA). Fetal bovine serum (FBS), Roswell Park Memorial Institute (RPMI)-1640 medium, and penicillin-streptomycin were obtained from Gibco-BRL (Grand Island, NY, USA). Caspase activity assay kits were obtained from R\&D Systems (Minneapolis, MN,USA). Extracellular signal-regulated kinase (ERK)-specific inhibitor, PD98059, c-Jun N-terminal kinase (JNK)-specific inhibitor, SP600125, p38 mitogen-activated protein kinase (MAPK)-specific inhibitor, SB203580, and Akt inhibitor, LY290042, were purchased from Calbiochem (San Diego, CA, USA). The DNA staining kit (Cycletest ${ }^{\mathrm{TM}}$ Plus Kit) and the enhanced chemiluminescence (ECL) kit were purchased from Becton-Dickinson (San Jose, CA, USA) and Amersham Pharmacia Biotech (Arlington Heights, IL, USA), respectively.

Antibodies. Antibodies specific for Fas, Fas ligand (FasL), tumor necrosis factor-related apoptosis-inducing ligand (TRAIL), Bcl-2, Bcl-xL, Bax, Bad, Bid, X-linked inhibitor of apoptosis protein (XIAP), inhibitor of apoptosis protein (IAP)-1, IAP-2, survivin, caspase-3, -8 and -9 , poly(ADP-ribose) polymerases (PARP), $\beta$-catenin, and phospholipase $\mathrm{C} \gamma 1$ (PLC $\gamma 1$ ) were obtained from Santa Cruz Biotechnology Inc. (Santa Cruz, CA, USA). Antibodies specific for ERK, JNK, p38 MAPK, Akt, phospho (p)-ERK, p-JNK, p-38 MAPK and p-Akt were purchased from Cell Signaling Technology, Inc. (Beverly, MA, USA). Antibodies specific for death receptor (DR)4 and DR5 were obtained from Calbiochem. Anti-actin antibody was obtained from Sigma-Aldrich.

Preparation of an ethanol extract of D. morbifera stem bark. The stem bark of D. morbifera used in this research was obtained from Dongeui University Oriental Hospital (Busan, Korea). To prepare the ethanol extract of D. morbifera stem bark (EEDM), the dried stem was extracted twice with $70 \%$ ethanol (with $24 \mathrm{~h}$ reflux), and the extract was then concentrated under reduced pressure. The decoction was filtered, lyophilized, and stored at $4^{\circ} \mathrm{C}$ until use. The dried extract yield from the starting crude materials was $\sim 17.8 \%$ (w/w). The lyophilized powder was dissolved in dimethyl sulfoxide (DMSO), filtered through a $0.22-\mu \mathrm{m}$ syringe filter to create a stock solution, and then adjusted to final concentrations using complete RPMI-1640 medium.

Cell culture and MTT assay. U937 human leukemia cells, Chang liver cells, and WI-38 lung fibroblast cells were purchased from the American Type Culture Collection (Rockville, MD, USA) and maintained at $37^{\circ} \mathrm{C}$ in $95 \%$ humidified air and $5 \% \mathrm{CO}_{2}$ in RPMI-1640 medium that was supplemented with $10 \% \mathrm{FBS}$ and $1 \%$ penicillin/streptomycin. Inhibition of cell proliferation was determined using an MTT assay. Briefly, cells were seeded in 6-well plates and treated with various concentrations of EEDM for 12 and $24 \mathrm{~h}$. After treatment, the MTT working solution $(0.5 \mathrm{mg} / \mathrm{ml})$ was added to each well and incubated continuously at $37^{\circ} \mathrm{C}$ for $3 \mathrm{~h}$. The culture supernatant was removed from the wells, and DMSO was added to completely dissolve the formazan crystals. The absorbance of each well was measured at a $540 \mathrm{~nm}$ wavelength with an ELISA reader (Molecular Devices, Sunnyvale, CA, USA). Morphological observations of cultured cells were carried out with an inverted microscope (Carl Zeiss, Germany).

Nuclear staining with DAPI. For nuclear DAPI staining, the cells were harvested and washed with ice-cold phosphatebuffered saline (PBS) and fixed with $3.7 \%$ paraformaldehyde (Sigma-Aldrich) in PBS for $10 \mathrm{~min}$ at room temperature. The fixed cells were washed with PBS and stained with $2.5 \mu \mathrm{g} / \mathrm{ml}$ DAPI solution for $10 \mathrm{~min}$ at room temperature. The cells were then washed twice with PBS and analyzed with a fluorescence microscope (Carl Zeiss).

Detection of DNA fragmentation with gel electrophoresis. Following EEDM treatment, the cells were lysed in a buffer containing $10 \mathrm{mM}$ Tris- $\mathrm{HCl}, \mathrm{pH} 7.4,150 \mathrm{mM} \mathrm{NaCl}, 5 \mathrm{mM}$ EDTA, and $0.5 \%$ Triton X-100 for $1 \mathrm{~h}$ at room temperature. The lysates were vortexed and cleared by centrifugation at $19,000 \mathrm{x} \mathrm{g}$ for $30 \mathrm{~min}$ at $4^{\circ} \mathrm{C}$. A $25: 24: 1(\mathrm{v} / \mathrm{v} / \mathrm{v})$ equal volume of neutral phenol:chloroform:isoamyl alcohol (Sigma-Aldrich) was used to extract DNA in the supernatant, followed by electrophoretic analysis on $1.5 \%$ agarose gels containing $0.1 \mu \mathrm{g} / \mathrm{ml}$ ethidium bromide (EtBr; Sigma-Aldrich). 
Flow cytometric analysis for measuring the sub-G1 phase and MMP values. The cells were collected, washed with cold PBS, and fixed in $75 \%$ ethanol at $4^{\circ} \mathrm{C}$ for $30 \mathrm{~min}$. The DNA content of the cells was measured using a DNA staining kit according to the manufacturer's instructions. Then flow cytometric analyses were carried out using a flow cytometer, and the relative DNA content was determined using CellQuest software, with the presence of red fluorescence as a marker. The MMP values were determined with the dual-emission potential-sensitive probe, JC-1. The cells were collected and incubated with $10 \mu \mathrm{M} \mathrm{JC}-1$ for $20 \mathrm{~min}$ at $37^{\circ} \mathrm{C}$ in the dark. The cells were then washed once with PBS and analyzed with a flow cytometer (22).

Protein extraction and western blot analysis. Cells were lysed for $30 \mathrm{~min}$ with lysis buffer $(20 \mathrm{mM}$ sucrose, $1 \mathrm{mM}$ EDTA, $20 \mu \mathrm{M}$ Tris-Cl, pH 7.2, $1 \mathrm{mM}$ DTT, $10 \mathrm{mM} \mathrm{KCl}, 1.5 \mathrm{mM}$ $\mathrm{MgCl}_{2}, 5 \mu \mathrm{g} / \mathrm{ml}$ pepstatin A, $10 \mu \mathrm{g} / \mathrm{ml}$ leupeptin, and $2 \mu \mathrm{g} / \mathrm{ml}$ aprotinin) to prepare the total protein. The supernatants were collected, and the protein concentrations were determined using a Bio-Rad protein assay kit (Bio-Rad, Hercules, CA, USA). For western blot analysis, an equal amount of protein was subjected to electrophoresis on sodium dodecyl sulfate (SDS)-polyacrylamide gels and transferred to a nitrocellulose membrane (Schleicher \& Schuell, Keene, NH, USA) by electroblotting. Blots were probed with the desired antibodies for $1 \mathrm{~h}$, incubated with the diluted enzyme-linked secondary antibodies, and visualized with the ECL solution according to the recommended procedure.

Caspase activity assay. Caspase activity was determined with colorimetric assay kits, which use synthetic tetrapeptides [Asp-Glu-Val-Asp (DEAD) for caspase-3, Ile-Glu-Thr-Asp (IETD) for caspase-8, Leu-Glu-His-Asp (LEHD) for caspase-9] labeled with p-nitroaniline (pNA). Briefly, cells were lysed in the supplied lysis buffer according to the manufacturer's protocol. The supernatants were collected and incubated with the supplied reaction buffer containing DTT and DEAD-pNA, IETD-pNA, or LEHD-pNA as the substrate at $37^{\circ} \mathrm{C}$. The reactions were measured by changes in absorbance at $405 \mathrm{~nm}$ using a microplate reader.

Statistical analysis. All experiments are expressed as the means \pm standard deviations (SDs) of at least 3 separate tests. The Student's t-test was used for single-variable comparisons, and a p-value $<0.05$ was considered to indicate a statistically significant result.

\section{Results}

EEDM inhibits cell growth in U937 cells. To investigate whether EEDM inhibits U937 cell growth, the cells were treated with various concentrations $(0-200 \mu \mathrm{g} / \mathrm{ml})$ of EEDM for 12 and $24 \mathrm{~h}$, and viability was measured with an MTT assay. Compared to the untreated control, after treatment with EEDM, as indicated in Fig. 1A, EEDM had a strong inhibitory effect on cell proliferation of U937 cells in a dose- and time-dependent manner. However, EEDM had no antiproliferative effect on Chang normal liver and WI-38 lung-derived cell lines. Direct observation using an inverted microscope
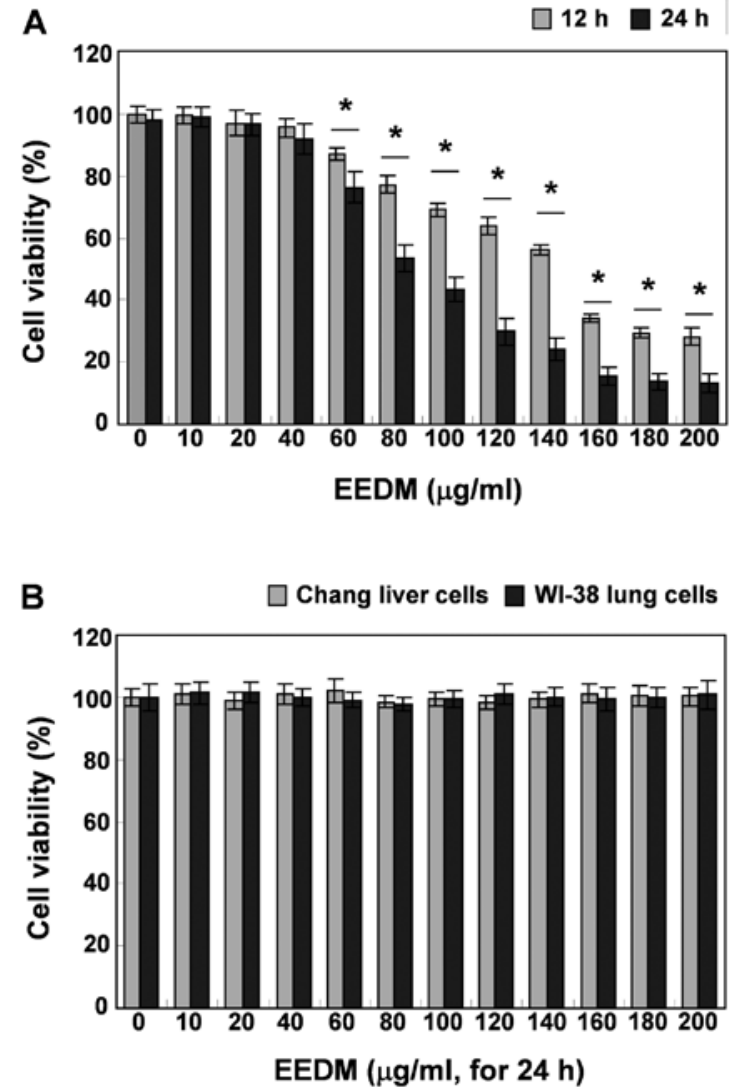

Figure 1. Effect of EEDM on the cell viability of (A) U937 cells and (B) Chang liver and WI-38 lung cells. Cell viability was measured using a metabolicdye-based MTT assay in cells after treatment with various concentrations of EEDM for 24 or 48 h. Cell viability under basal conditions (i.e. no EEDM) was considered to be $100 \%$. The data are expressed as the means \pm SD of triplicate samples. ${ }^{*} \mathrm{p}<0.05$, significant difference vs. control cells. EEDM, ethanol extract of D. morbifera stem bark.

showed that numerous morphological changes occurred in the U937 cells treated with EEDM. In particular, cell shrinkage and cytoplasmic condensation appeared in a concentrationdependent manner after EEDM treatment (Fig. 2A).

EEDM induces apoptosis in U937 cells. Further experiments were conducted to determine whether the inhibition of cell viability observed in the presence of EEDM was the result of apoptotic cell death in U937 cells. To examine the apoptosis morphologically, the nuclei of untreated and EEDM-treated cells were stained with DAPI solution and then observed. Following treatment of U937 cells with various concentrations of EEDM for $24 \mathrm{~h}$, the chromatin stained with DAPI had a characteristic condensed and fragmented appearance in a concentration-dependent fashion (Fig. 2B). In addition, following agarose gel electrophoresis of the DNA from cells treated with EEDM, a characteristic ladder pattern of discontinuous DNA fragments was observed; however, DNA fragmentation was barely detected in the control cells (Fig. 2C). We next quantified the apoptotic dead cells using a flow cytometer. As shown in Fig. 2D, EEDM treatment resulted in a significant increase in the number of U937 cells in the apoptotic sub-G1 phase, and this response occurred in a concentration-dependent manner. These results confirmed 


\section{A}

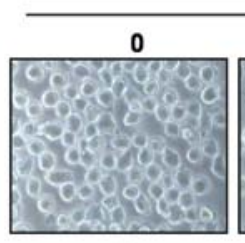

60

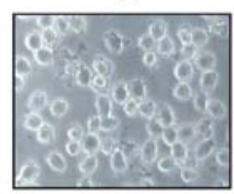

B

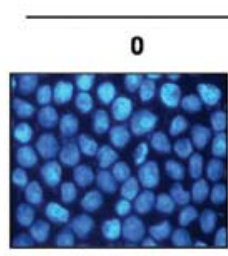

60
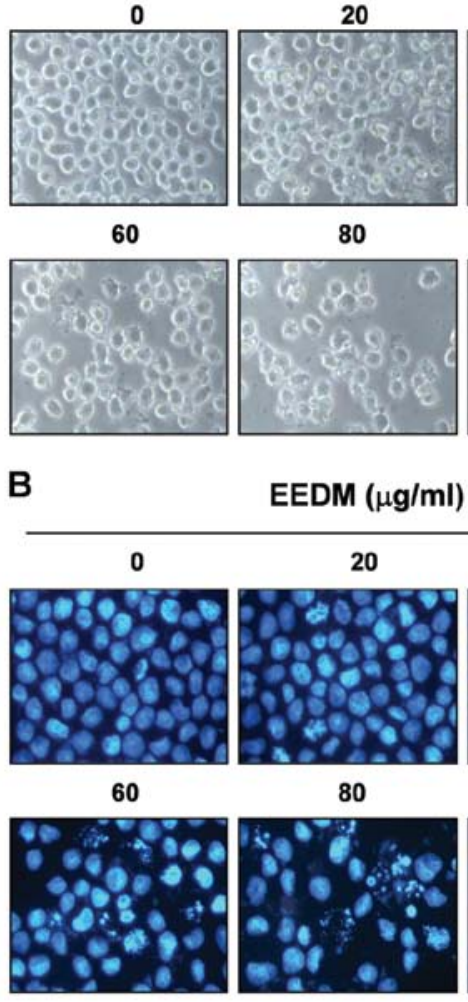

80

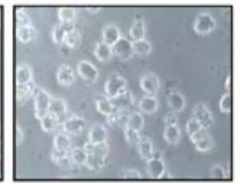

$\operatorname{EEDM}(\mu \mathrm{g} / \mathrm{ml})$

20

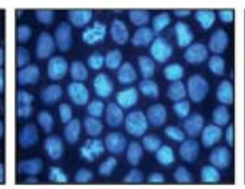

80
$\operatorname{EEDM}(\mu \mathrm{g} / \mathrm{ml})$

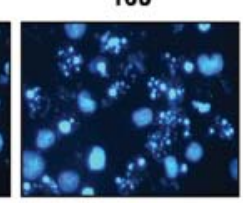

C

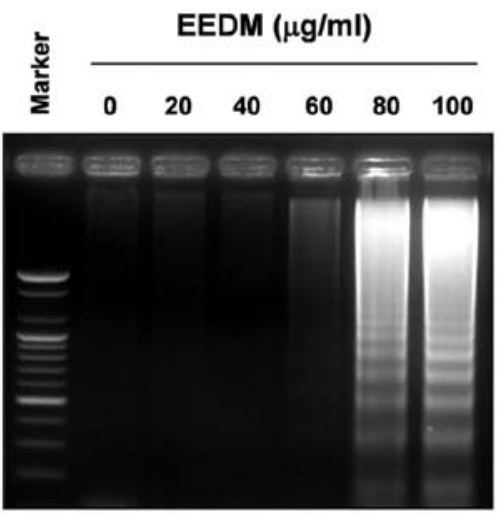

D

$\operatorname{EEDM}(\mu \mathrm{g} / \mathrm{ml})$

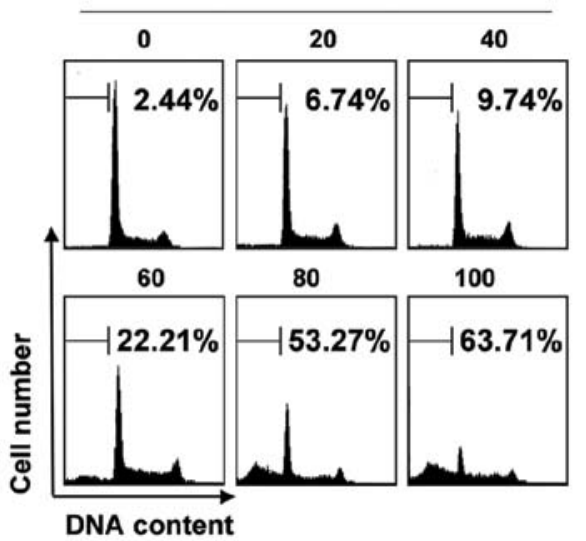

Figure 2. Induction of apoptosis by EEDM treatment in U937 cells. U937 cells were exposed to the indicated concentrations of EEDM for 24 h. (A) The morphology of the cells was imaged using an inverted microscope (original magnification, x200). (B) The cells were fixed and stained with DAPI solution. After a 10-min incubation at room temperature, stained nuclei were observed with a fluorescence microscope (original magnification, $\mathrm{x} 400$ ). (C) For the DNA fragmentation analysis, genomic DNA was extracted, separated by $1.5 \%$ agarose gel electrophoresis, and visualized under UV light after staining with EtBr. The marker indicates a size marker of the DNA ladder. The results shown are from one representative experiment of 2 experiments that showed similar patterns. (D) To quantify the degree of apoptosis induced by EEDM, the DNA content was analyzed using a flow cytometer, and the percentage of sub-G1 phase cells was determined based on a DNA content histogram. Data are the mean of 2 different experiments. EEDM, ethanol extract of $D$. morbifera stem bark.

A

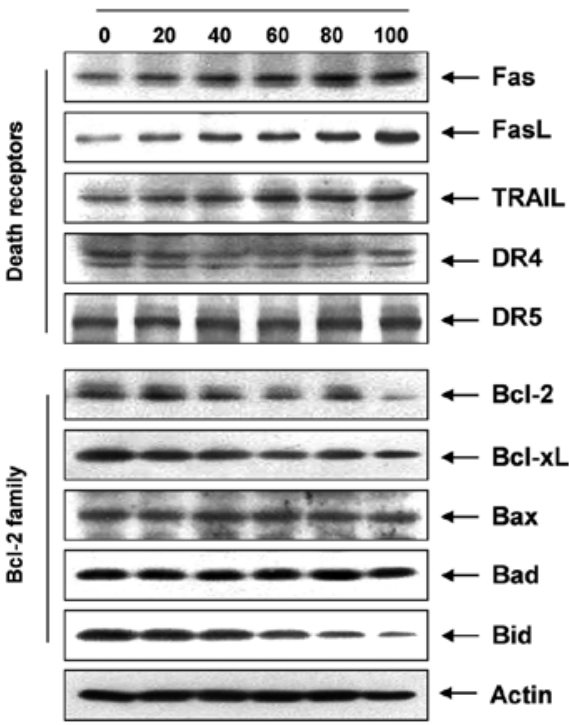

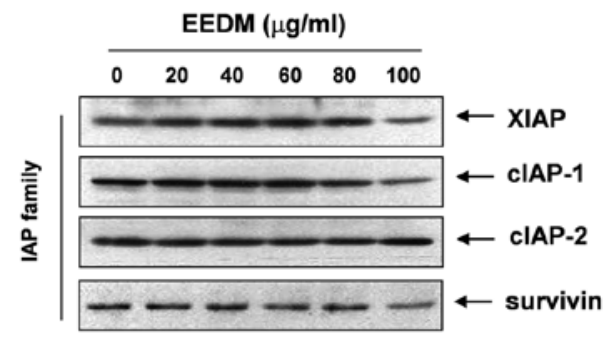

B

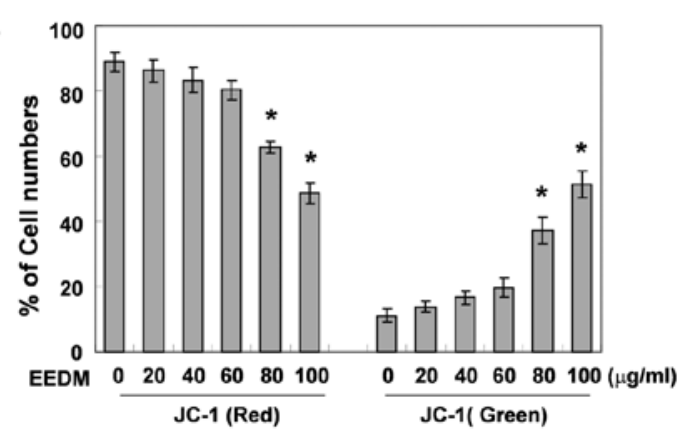

Figure 3. Effects of EEDM on levels of apoptosis-related proteins and MMP values in U937 cells. U937 cells were treated with the indicated concentrations of EEDM for $24 \mathrm{~h}$. (A) The cell lysates were separated with SDS-polyacrylamide gel electrophoresis, and western blot analyses were performed using the indicated antibodies and an ECL detection system. Actin was used as an internal control. (B) The cells were stained with JC-1, and the mean JC-1 fluorescence intensity was detected using a flow cytometer. The data are expressed as the means \pm SD of triplicate samples. " $\mathrm{p}<0.05$, significant difference vs. control cells. EEDM, ethanol extract of D. morbifera stem bark. 
that EEDM inhibits the proliferation of U937 cells by inducing apoptosis.

EEDM alters the expression of apoptosis-related proteins and MMP values in U937 cells. To investigate the mechanisms underlying EEDM-induced apoptosis, the expression level of several apoptotic factors was analyzed. Fig. 3A demonstrates that, after the cells were exposed to EEDM, the levels of the death receptor-related proteins Fas, FasL and TRAIL, but not DR4 and DR5, were concentration-dependently increased. Fig. 3A also shows that the levels of the anti-apoptotic Bcl-2 family of proteins (Bcl-2 and Bcl-xL) were decreased in response to EEDM; however, the levels of pro-apoptotic Bax and Bad remained unchanged. In addition, although we did not detect the truncated form of the pro-apoptotic protein Bid, EEDM decreased the total form of the Bid proteins, reflecting Bid cleavage and activation. Under these conditions, levels of the anti-apoptotic IAP family of proteins such as XIAP, cIAP-1 and survivin, which bind to caspases and lead to their inactivation, were inhibited by EEDM treatment in a concentration-dependent manner.

EEDM induces the loss of MMP. Mitochondrial membrane permeabilization, accompanied by the collapse of the electrochemical gradient across the mitochondrial membrane, is a key event during cellular apoptosis. To investigate whether mitochondrial dysfunction was involved in EEDM-induced U937 cell apoptosis, we used flow cytometric analysis with JC-1 staining to examine the change in the MMP values after EEDM treatment. JC-1 is a lipophilic and cationic dye that selectively enters mitochondria. In healthy cells with high mitochondrial potential, JC-1 forms J-aggregates with intense red fluorescence $(590 \mathrm{~nm})$, whereas under apoptotic conditions, the mitochondrial membrane potential collapses. Thus, JC-1 does not accumulate within the mitochondria but remains in the cytoplasm in a monomeric form showing green fluorescence (525 nm). As shown in Fig. 3B, JC-1 fluorescence shifted from a JC-1-red-bright/JC-1-green-bright signal in the control cells to a JC-1-green-bright/JC-1-red-dim signal in the EEDM-treated cells in a dose-dependent manner, indicating EEDM induced loss of the MMP in U937 cells.

EEDM induces activation of caspases in U937 cells. To investigate the apoptotic cascade induced by EEDM, U937 cells were exposed to various concentrations of EEDM for $24 \mathrm{~h}$, after which the expression level and activity of caspase-3, -8 and -9 were measured. As shown in Fig. 4, the expression of pro-caspase-3,-8 and -9 decreased following EEDM treatment, which was associated with a significant concentration-dependent increase in the activity of these caspases compared with the untreated control cells. Furthermore, subsequent western blot analysis revealed that EEDM induced proteolytic degradation of PARP, $\beta$-catenin, and PLC $\gamma 1$, downstream target proteins of activated caspase-3. Cleavage fragments of these proteins gradually increased over time.

To confirm the role of caspase activation in EEDM-induced apoptosis, U937 cells were exposed to a broad-spectrum caspase inhibitor, $\mathrm{z}-\mathrm{VED}$-fmk, for $2 \mathrm{~h}$ before EEDM was added. As shown in Fig. 5, pretreatment with z-VED-fmk significantly attenuated EEDM-induced chromatin condensation, the
A

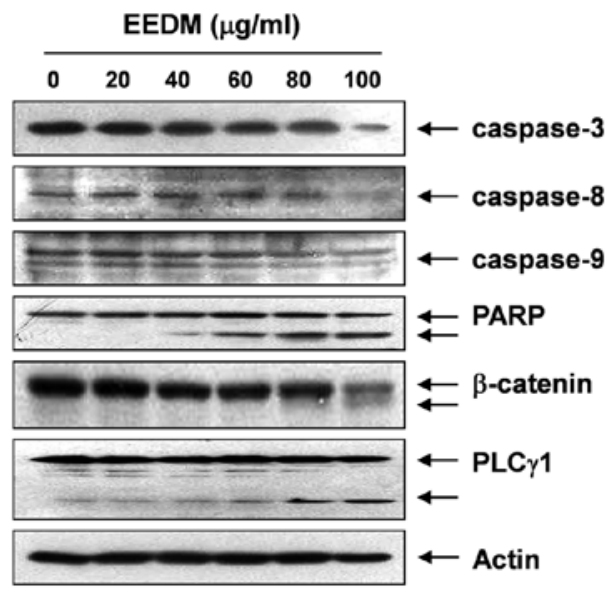

B

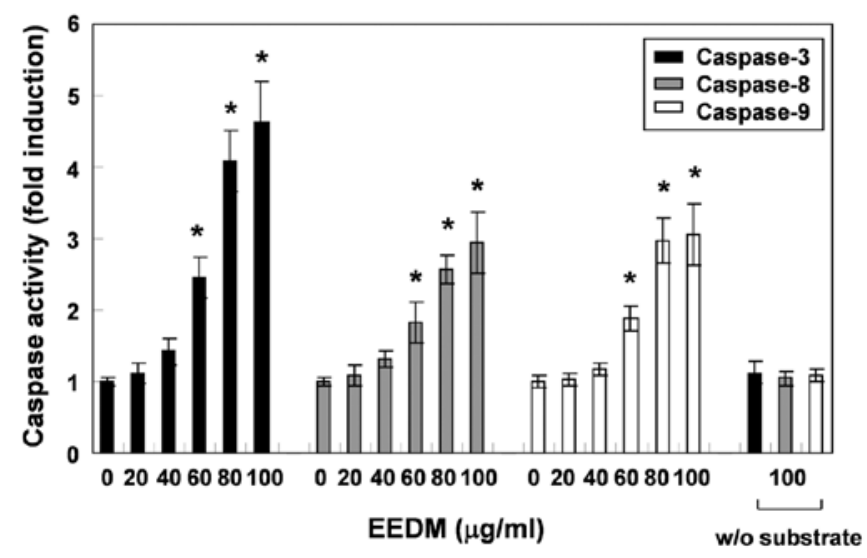

Figure 4. Caspase activation and degradation of caspase-3 substrate proteins by EEDM treatment in U937 cells. Cells were incubated in the presence or absence of several concentrations of EEDM for $24 \mathrm{~h}$. (A) Cells were subjected to western blot analysis using the indicated antibodies and an ECL detection system. Actin was used as an internal control. (B) Caspase-3, -8, and -9 activity was determined with colorimetric assay kits. The data were normalized to the caspase activity within the control cells and are represented as the fold of the control. Data are averages with SD from at least 3 independent experiments. " $\mathrm{p}<0.05$, significant difference vs. control cells. EEDM, ethanol extract of D. morbifera stem bark.

formation of apoptotic bodies, and DNA fragmentation and restored the increased apoptosis. Furthermore, z-VED-fmk alone did not affect cell viability but reversed the anti-proliferative activity of EEDM (Fig. 5C). These data clearly indicate that EEDM-induced apoptosis in U937 cells was mediated by caspase activation.

Effects of EEDM on the MAPK and PI3K/Akt signaling pathways in U937 cells. To address whether the activation of the MAPK signaling pathway is involved in EEDM-induced apoptosis, we investigated whether members of the MAPK family of proteins are activated during EEDM-induced apoptosis. As Fig. 6A demonstrates, stimulation of U937 cells with EEDM led to rapid phosphorylation of MAPKs, including p38 MAPK, ERK and JNK, with peak levels of each phospho-MAPK observed 0.5 to $6 \mathrm{~h}$ after EEDM was added. However, the specific inhibitors of each protein (PD98059, ERK-specific inhibitor; SP600125, JNK-specific inhibitor and SB203580, p38 MAPK-specific inhibitor) did not block EEDM-induced cell death (Fig. 6B), indicating that EEDM-induced apoptosis 
A

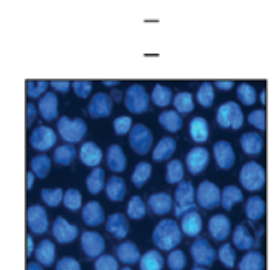

B

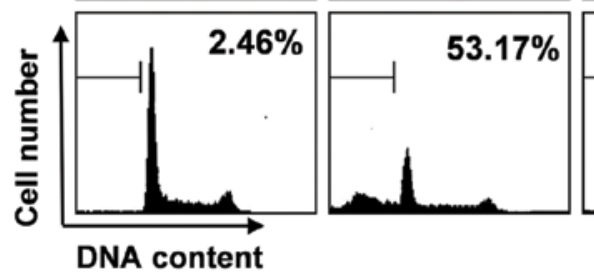

-.
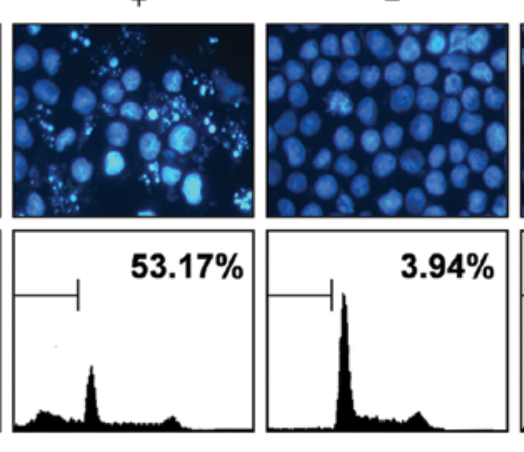

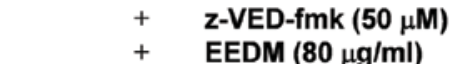

$+\quad$ EEDM $(80 \mu \mathrm{g} / \mathrm{ml})$
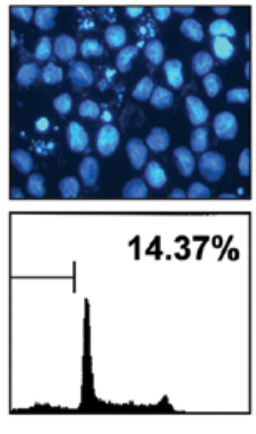

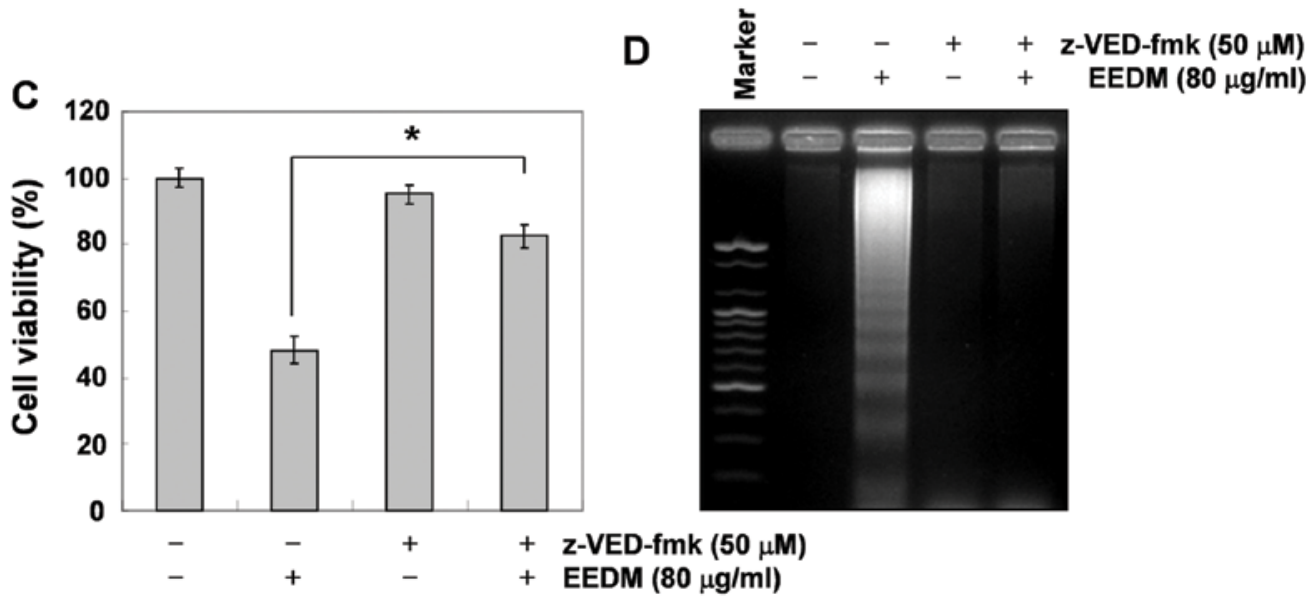

Figure 5. Effects of a pan-caspase inhibitor on the apoptosis induced by EEDM in U937 cells. (A) Cells were incubated in the presence or absence of $\mathrm{z}$-VED-fmk for $2 \mathrm{~h}$ before being exposed to EEDM. After $24 \mathrm{~h}$ of incubation, the cells were fixed, stained with DAPI solution, and then observed under a fluorescence microscope (original magnification, x400). (B) Cells grown under the same conditions as (A) were used to evaluate the sub-G1 DNA content using a flow cytometer. Data are the mean of 2 different experiments. (C) Cell viability was analyzed using an MTT assay. The data are expressed as the means \pm SD of triplicate samples. ${ }^{*}$ p $<0.05$, significant difference vs. EEDM-treated cells. (D) DNA fragmentation was analyzed using a $1.5 \%$ agarose gel for electrophoresis. EEDM, ethanol extract of D. morbifera stem bark.

is independent of the MAPK pathway. We next examined the involvement of the phosphatidylinositol-3-kinase (PI3K)/ Akt pathway in EEDM-induced U937 apoptosis. As shown in Fig. 6A, the levels of phosphorylated Akt, a downstream kinase of PI3K, were time-dependently decreased in response to EEDM, while the total Akt protein levels remained constant during EEDM treatment. Similar to MAPK inhibitors, combination treatment with EEDM and LY294002, a specific inhibitor of PI3K, did not affect EEDM-induced apoptosis. These results suggest that EEDM-induced apoptosis is not associated with downregulation of the Akt signaling pathway.

\section{Discussion}

The induction of apoptotic cell death is a promising emerging strategy for the treatment of cancer, and many studies have screened for pro-apoptotic natural compounds. Moreover, natural products, such as traditional herbal medicines, have relatively fewer side-effects compared to modern chemotherapeutics and have long been used clinically to treat various diseases, including cancer. Therefore, discovering naturally occurring agents with pro-apoptotic activity in cancer cells is a promising approach for developing novel cancer chemotherapies. Although the findings from recent studies have demonstrated that the components of D. morbifera have diverse pharmacological properties, including anti-inflammatory and anticancer effects (14-21), the mode of action of its antitumor property is still largely unknown. Therefore, before the extract of D. morbifera can be further developed as anticancer agent, the antitumor activity and the underlying molecular mechanism must be elucidated. The results of this study clearly demonstrate that EEDM inhibited U937 leukemic cell growth, but not normal cells, by induction of apoptosis. After the cells were exposed to EEDM, chromatin condensation, apoptotic bodies and DNA fragmentation were clearly observed. Furthermore, the data obtained from flow cytometric analysis after PI staining confirmed EEDM-induced apoptosis in U937 cells (Figs. 1 and 2).

Apoptosis manifests as two major pathways that initiate apoptosis designated as intrinsic (i.e. mitochondrial) and extrinsic (i.e. death receptor) pathways. The intrinsic pathway is controlled by the Bcl-2 family proteins, which play important regulatory roles in apoptosis, by either inhibiting or promoting apoptosis $(1,2)$. Heterodimerization between the pro- (such as Bcl-2 and Bcl-xL) and anti-apoptotic proteins (such as Bax and $\mathrm{Bad}$ ) of this family and the balance of Bcl-2 family members may determine the susceptibility to a given apoptotic stimulus and cell fate (23). In the extrinsic pathway, the roles of death 
A
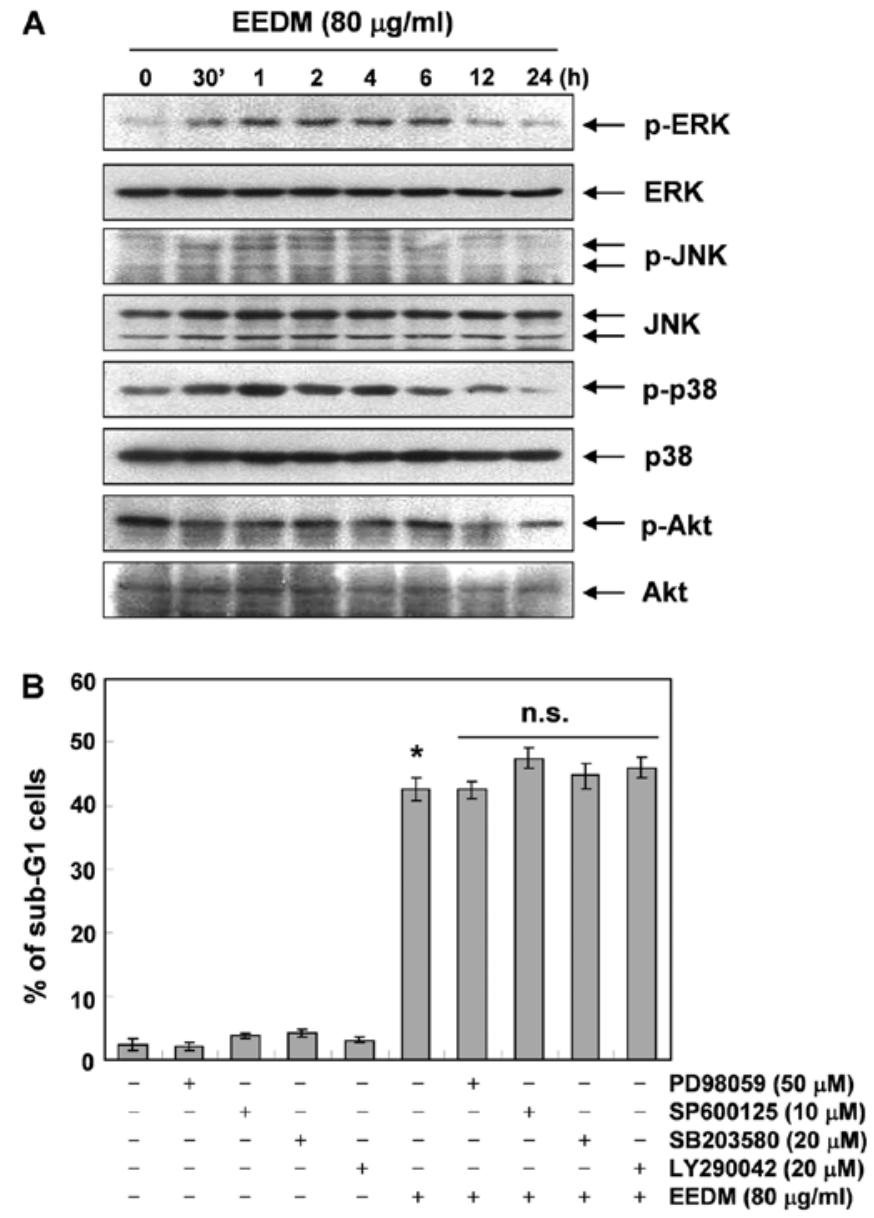

Figure 6. Involvement of MAPK and PI3K/Akt signaling pathways on EEDM-induced apoptosis in U937 cells. (A) Cells were treated with $80 \mu \mathrm{g} / \mathrm{m}$ EEDM for the indicated times. Cells were then lysed, and equal amounts of cell lysates were resolved on SDS-polyacrylamide gels, transferred to nitrocelluloses, and probed with the indicated antibodies. (B) Cells were pretreated with the indicated MAPK inhibitors (SB203580, $20 \mu \mathrm{M}$; SP600125 $20 \mu \mathrm{M}$; and PD98059, $50 \mu \mathrm{M})$ or Akt inhibitor (10 $\mu \mathrm{M} \mathrm{LY} 290042)$ for $1 \mathrm{~h}$ and then treated with $80 \mu \mathrm{g} / \mathrm{ml}$ EEDM for $24 \mathrm{~h}$. The percentage of the sub-G1 population was evaluated with a flow cytometer. The data are expressed as the means \pm SD of triplicate samples. ${ }^{*} \mathrm{p}<0.05$, significant difference vs. control cells; n.s., not significant vs. EEDM-treated cells. EEDM, ethanol extract of D. morbifera stem bark.

legends and their receptors are well-characterized. When death legends engage death receptors, a protein complex forms, and caspase- 8 activation leads to apoptosis $(2,12)$. Apoptosis may also be inhibited by various proteins, including members of the IAP family, which are largely overexpressed by most tumors. These proteins promote tumor cell survival due to direct inhibition by binding to several caspases after a wide variety of apoptotic stimuli elicited via the intrinsic and extrinsic pathways $(24,25)$. In this study, EEDM did not alter the expression of pro-apoptotic Bax and Bad in U937 cells but downregulated the expression of anti-apoptotic $\mathrm{Bcl}-2$ and $\mathrm{Bcl}-\mathrm{xL}$, resulting in an increase in the ratio of Bax/ Bad:Bcl-2 and Bax/Bad:Bcl-xL. Additionally, an increase in the Fas, FasL and TRAIL proteins, crucial members of the extrinsic pathway, was observed in the EEDM-treated U937 cells (Fig. 3). Our results also revealed that EEDM-induced apoptosis was related to downregulation of IAP family proteins such as XIAP, cIAP-1 and survivin (Fig. 3). These results indicate that EEDM-induced apoptosis correlates strongly with the downregulation of anti-apoptotic proteins.

Cellular demolition in apoptosis is carried out by caspases, which are key executioners of apoptosis and play important roles in drug-induced apoptosis in a large variety of cancer cells. Two members of this group of enzymes are known as 'initiator' and 'effector' caspases $(5,7)$. Upon activation, extrinsic and intrinsic caspases such as caspase-8 and -9, respectively, trigger the proteolytic activation of executioner caspases such as caspase-3 and -7. In general, caspase- 3 is the common effector for most apoptotic pathways, and its active form is responsible for the cleavage and breakdown of several cellular components related to DNA repair and regulation. Once activated, caspase-3 also cleaves numerous important cellular substrates and causes membrane blebbing, disassembly of the cell structure, and DNA fragmentation, which eventually lead to cell death (8-10). In addition, activated caspase- 8 can convert Bid to truncated Bid (tBid), leading to mitochondrial depolarization and the release of many apoptogenic proteins, such as cytochrome $c$, from the mitochondrial intermembrane space after translocation of tBid to the mitochondria (11). This leads finally to the activation of caspase- 3 and induction of apoptosis via a complex of apoptotic protease activating factor-1 (Apaf-1), pro-caspase-9, and cytochrome $c(2,12)$. During the process, the electrochemical gradient across the mitochondrial membrane collapses; therefore, the loss of MMP is another hallmark of apoptosis. Our data clearly showed that treatment with EEDM led to the collapse of the MMP, which was associated with the reduction of whole Bid proteins, which may relate to the activation of tBid (Fig. 3). The data also revealed that exposing U937 cells to EEDM resulted in the proteolytic activation of caspase- 8 and -9 , which involves initiator caspases of the extrinsic and intrinsic pathways, respectively (Fig. 4), suggesting that extrinsic and intrinsic pathways may have contributed, at least in part, to EEDM-induced apoptosis of U937 cells. Furthermore, EEDM markedly activated the key executioner, caspase-3, and the concomitant degradation of PARP, $\beta$-catenin, and PLC $\gamma 1$ proteins (Fig. 4). However, under the same experimental conditions, significant protection of EEDM-induced apoptosis was observed following pretreatment with a pan caspase inhibitor, z-VED-fmk (Fig. 5). These data indicate that caspases are the key molecules mediating EEDM-induced apoptosis, and EEDM-induced apoptosis in U937 cells may be mediated through a caspase-dependent pathway.

In contrast, the PI3K/Akt and MAPK signaling pathways play critical roles in cell survival and apoptosis in various cancer cells. The activation of PI3K/Akt and ERK pathways leads to cell survival through the phosphorylation of various anti-apoptotic downstream effectors, whereas the p38 MAPK and JNK pathways are more often associated with the induction of apoptosis (26-29). Our data indicated that phosphorylation of all three MAPKs increased rapidly in U937 cells in response to EEDM and peaked after 0.5 to $6 \mathrm{~h}$; whereas, phosphorylation of Akt, downstream of PI3K, decreased in response to EEDM treatment after $12 \mathrm{~h}$. However, all specific inhibitors of the PI3K/Akt and MAPK signaling pathways did not inhibit EEDM-induced apoptosis of U937 cells (Fig. 6). Although more experiments are required to find the relationship between 
EEDM and these signaling pathways, the results indicate that EEDM-induced apoptosis in U937 cells is not associated with the PI3K/Akt and MAPK signaling pathways.

In conclusion, the present study clearly demonstrates that EEDM significantly inhibits cell proliferation and induces apoptosis in U937 cells via the extrinsic and intrinsic pathways. EEDM-induced apoptosis was mediated by caspase activation and was triggered by mitochondrial dysfunction and modulation in Bcl-2 and IAP family protein levels. These novel phenomena have not been previously described and provide important new insights into the biological effects of EEDM. These results warrant further investigation of $D$. morbifera as a source of pharmacologically active agents as well as the identification and purification of the active compounds in the present EEDM. In vivo studies using preclinical tumor models are also needed to fully establish the potential of EEDM as a chemopreventive and therapeutic agent in cancer.

\section{Acknowledgements}

This research was supported by the Basic Science Research Program through the National Research Foundation of Korea grant funded by the Korean government (no. 2012046358).

\section{References}

1. Jin Z and El-Deiry WS: Overview of cell death signaling pathways. Cancer Biol Ther 4: 139-163, 2005.

2. Fulda S and Debatin KM: Extrinsic versus intrinsic apoptosis pathways in anticancer chemotherapy. Oncogene 25: 4798-4811, 2006.

3. Brenner D and Mak TW: Mitochondrial cell death effectors. Curr Opin Cell Biol 21: 871-877, 2009.

4. Röder C, Trauzold A and Kalthoff H: Impact of death receptor signaling on the malignancy of pancreatic ductal adenocarcinoma. Eur J Cell Biol 90: 450-455, 2011.

5. Fulda S and Kroemer G: Mitochondria as therapeutic targets for the treatment of malignant disease. Antioxid Redox Signal 15: 2937-2949, 2011.

6. Kim IH, Kim SW, Kim SH, Lee SO, Lee ST, Kim DG, Lee MJ and Park WH: Parthenolide-induced apoptosis of hepatic stellate cells and anti-fibrotic effects in an in vivo rat model. Exp Mol Med 44: 448-456, 2012.

7. Ola MS, Nawaz M and Ahsan H: Role of Bcl-2 family proteins and caspases in the regulation of apoptosis. Mol Cell Biochem 351: 41-58, 2001.

8. Lazebnik YA, Kaufmann SH, Desnoyers S, Poirier GG and Earnshaw WC: Cleavage of poly(ADP-ribose) polymerase by a proteinase with properties like ICE. Nature 371: 346-347, 1994.

9. Kwon TK: Phorbol myristate acetate inhibits okadaic acidinduced apoptosis and downregulation of X-linked inhibitor of apoptosis in U937 cells. Biochem Biophys Res Commun 287: $135-141,2001$.

10. Fukuda K: Apoptosis-associated cleavage of $\beta$-catenin in human colon cancer and rat hepatoma cells. Int J Biochem Cell Biol 31 519-529, 1999.
11. Eskes R, Desagher S, Antonsson B and Martinou JC: Bid induces the oligomerization and insertion of Bax into the outer mitochondrial membrane. Mol Cell Biol 20: 929-935, 2000.

12. Shamas-Din A, Brahmbhatt H, Leber B and Andrews DW: BH3-only proteins: orchestrators of apoptosis. Biochim Biophys Acta 1813: 508-520, 2011.

13. Newman DJ and Cragg GM: Natural products as sources of new drugs over the 30 years from 1981 to 2010. J Nat Prod 75: 311-335, 2012.

14. Moon HI: Antidiabetic effects of dendropanoxide from leaves of Dendropanax morbifera Leveille in normal and streptozotocininduced diabetic rats. Hum Exp Toxicol 30: 870-875, 2011.

15. Chung IM, Kim MY, Park WH and Moon HI: Antiatherogenic activity of Dendropanax morbifera essential oil in rats. Pharmazie 64: 547-549, 2009.

16. Chung IM, Kim MY, Park SD, Park WH and Moon HI: In vitro evaluation of the antiplasmodial activity of Dendropanax morbifera against chloroquine-sensitive strains of Plasmodium falciparum. Phytother Res 23: 1634-1637, 2009.

17. Chung IM, Song HK, Kim SJ and Moon HI: Anticomplement activity of polyacetylenes from leaves of Dendropanax morbifera Leveille. Phytother Res 25: 784-786, 2011.

18. Park BY, Min BS, Oh SR, Kim JH, Kim TJ, Kim DH, Bae KH and Lee HK: Isolation and anticomplement activity of compounds from Dendropanax morbifera. J Ethnopharmacol 90: 403-408, 2004.

19. Richmond JD, Agius BR, Wright BS, Haber WA, Moriarity DM and Setzer WN: Essential oil compositions and cytotoxic activities of Dendropanax capillaris, Oreopanax nubigenus, and Schefflera rodrigueziana from Monteverde, Costa Rica. Nat Prod Commun 4: 271-274, 2009.

20. Yu HY, Kim KS, Lee YC, Moon HI and Lee JH: Oleifolioside A, a new active compound, attenuates LPS-stimulated iNOS and $\mathrm{COX}-2$ expression through the downregulation of NF- $\kappa \mathrm{B}$ and MAPK activities in RAW 264.7 macrophages. Evid Based Complement Alternat Med 2012: 637512, 2012.

21. Yu HY, Jin CY, Kim KS, Lee YC, Park SH, Kim GY, Kim WJ, Moon HI, Choi YH and Lee JH: Oleifolioside A mediates caspase-independent human cervical carcinoma HeLa cell apoptosis involving nuclear relocation of mitochondrial apoptogenic factors AIF and EndoG. J Agric Food Chem 60: 5400-5406, 2012.

22. Tak JK, Lee JH and Park JW: Resveratrol and piperine enhance radiosensitivity of tumor cells. BMB Rep 45: 242-246, 2012.

23. Nechushtan A, Smith CL, Hsu YT and Youle RJ: Conformation of the Bax C-terminus regulates subcellular location and cell death. EMBO J 18: 2330-2341, 1999.

24. Danson S, Dean E, Dive C and Ranson M: IAPs as a target for anticancer therapy. Curr Cancer Drug Targets 7: 785-794, 2007.

25. Altieri DC: Survivin and IAP proteins in cell-death mechanisms. Biochem J 430: 199-205, 2010.

26. Xia Z, Dickens M, Raingeaud J, Davis RJ and Greenberg ME: Opposing effects of ERK and JNK-p38 MAP kinases on apoptosis. Science 270: 1326-1331, 1995.

27. Ashkenazi A: Targeting the extrinsic apoptosis pathway in cancer. Cytokine Growth Factor Rev 19: 325-331, 2008.

28. Aksamitiene E, Kiyatkin A and Kholodenko BN: Cross-talk between mitogenic Ras/MAPK and survival PI3K/Akt pathways: a fine balance. Biochem Soc Trans 40: 139-146, 2012.

29. Boutros T, Chevet E and Metrakos P: Mitogen-activated protein (MAP) kinase/MAP kinase phosphatase regulation: roles in cell growth, death, and cancer. Pharmacol Rev 60: 261-310, 2008. 